\title{
Towards an Environmental Management Approach for Collaborative Networks
}

\author{
Ovidiu Noran \\ Griffith University, Australia \\ O.Noran@griffith.edu.au
}

\begin{abstract}
Environmental responsibility is evolving into a crucial aspect of strategic management as regulations are expected to tighten significantly in the near future following the need to mitigate the effects of climate change. Many businesses react to this challenge by implementing environmental reporting and management systems. However, often the environmental programme is not properly integrated in the overall business strategy and the management does not have timely access to appropriate environmental information. Moreover, within collaborative networks (CNs) the environmental management (EM) effort should be coordinated amid partners to achieve a much needed synergy and also enable prompt deployment of the required EM artefacts within the virtual organisations (VOs) created. This paper attempts to show a possible way to integrate environmental management (EM) into the VOs set up by the $\mathrm{CN}$ participants by using an Enterprise Architecture (EA) life cycle-based approach.
\end{abstract}

Keywords: Environmental management, enterprise architecture.

\section{Introduction}

The continued existence of businesses depends not only on their economic sustainability but also on their impact on the natural environment and on the way they treat their workers. This fact was emphasized by Elkington's [1] Triple Bottom Line (TBL) approach to business sustainability, stating that one must achieve not only economic bottom-line performance but also environmental / social accomplishment. Thus, Blackburn [2] compares economic sustainability to air and environmental and social sustainability to food: the first is more urgent however not more important than the second. The '2Rs' (Respect for humans and judicious Resource management) are another essential component of overall sustainability of a business. Hence, successful enterprises must take a whole-system approach to sustainable development (see [3]).

The above requirements add a new degree of complexity to the management of Collaborative Networks (CNs), which are typically formed so as allow their members to promptly create virtual organisations (VOs) that are able to bid for projects that go beyond the individual competencies of the CN participants [4].

This paper focuses on the challenges presented by the proper integration of the environmental sustainability aspect in the VOs created by a CNO and proposes a possible way to address these challenges based on an Enterprise Architecture (EA) approach. 


\section{Environmental Management: Is There a Better Way?}

Currently, EM efforts within many companies are fairly incoherent and often not properly supported by the ICT infrastructure. Thus, business units may approach environmental sustainability in different levels of detail and at a different pace producing a possible loss of aggregate capabilities; moreover, often the management cannot effectively use the information generated by the environmental reporting functions due to language, format, level of aggregation etc. Strategic EM integration requires prompt and effective access to environmental information facilitating the decision-making process $[5,6]$. In the case of CNs, this challenge is complicated by the need to mediate between- and coordinate the EM approaches of the participants in order to promptly construct and deploy agreed-upon EM frameworks for the VOs created. An optimal way to solve this problem would be to set up an EM project with a) top-management support for the project champion(s), b) sufficient authority for the manager and appropriate human / infrastructure resources, c) a plan that is integrated in the general company strategic direction and d) a cross-departmental approach.

These requirements match to a good extent the scope of typical EA projects; it is therefore proposed here that EA could provide a solution to an integrated approach to the coordination of environmental aspects in the management and operation of the $\mathrm{CN}$ participants and especially their prompt introduction in the VOs created and operated by the $\mathrm{CN}$. This is desirable because a company whose architecture includes EM competencies and responsibilities in an integrated fashion will have the necessary agility and preparedness required from $\mathrm{CN}$ participants and from the $\mathrm{VO}$ in particular.

\section{Brief Review of Relevant Environmental Management Artefacts}

Many companies address the need to introduce environmental responsibility in their business units by attempting to implement some type of environmental reporting and environmental management system (EMS).

While an EMS is a step in the right direction, when implemented in isolation it may not trigger the cultural change necessary to achieve permanent environmental responsibility. Thus, Coglianese and Nash [7] argue that the implementation of an EMS alone is irrelevant in the absence of genuine commitment to environmental improvements. It must be noted that relevant regulation such as ISO 14001:2004 [8] limits the constraints on an EMS to being designed such that companies can work towards the goal of regulatory compliance and seek to make improvements; it does not actually require achieving actual compliance with existing law.

Various reference models for EMS design and EMS alternatives have emerged. For example, Blackburn [2] proposes to rather build a 'Sustainability Operating System' i.e. a management method to achieve sustainability based on the Brundtland report [3], the '2R's and the TBL approach applied to sustainability. Willard [9] recommends a TBL-based approach encompassing economy / profit, environment / planet and equity / people with obvious benefits: easier hiring and retention, increased productivity, reduced manufacturing / commercial site expenses, increased revenue / market share and reduced risk. Clayton and Redcliffe [10] advocate a systems 
approach towards the integration of sustainability aspects into the business and define the environmental quality as capital (and thus the viability of 'tradable pollution').

EM frameworks aim to provide a structured set of artefacts (methods, aspects, reference models, etc) specialised for the EM area. Some examples: The Natural Step (TNS) Framework, proposing a systems-based approach to organisational planning for sustainability [11], The Natural Edge Project [12] with a holistic approach taking into account system life cycle and the Life Cycle Management Framework for continuous environmental improvement [13]. Assessment and reporting frameworks aim to assist the measurement and reporting functions of the EMS. For example, the Life Cycle Assessment (LCA) method measures the environmental impacts of products or services relative to each other during their life cycles [14]. The Global Reporting Initiative's sustainability reporting framework [15] contains reporting principles and guidance potentially applicable to all types of businesses.

International Standards also cover the EM issue. ISO 14000:2004 is a set of reference models for EMS setup, life-cycle assessment, environmental auditing of processes and environmental labelling and performance evaluation. ISO 14001:2004 deals specifically with EMS-s, aiming to provide a framework for a holistic and strategic approach to the organization's environmental policy, plans and actions [8]. Standards provide a good starting point for design and assessment; unfortunately however, as mentioned current EM standards do not enforce EM performance levels.

As a conclusion, many of the above-mentioned artefacts recognize the need to analyse the life cycle of the products. However, in reality it is often required to also take into account other life cycles as well. For example in a scenario of a VO created by a $\mathrm{CN}$ one must consider the life cycles of the $\mathrm{CN}$ participants, of the $\mathrm{VO}$, of the project to create the VO's EMS and of course the life cycle of the EMS itself. It is also necessary to analyse the interactions between these entities in that context. The proposed approach involves life cycle architectures elements that describe systems during their entire life and thus allow identifying potential problems and aspects that may not be otherwise obvious in a snapshot-type representation.

\section{Tools Used in the Proposed Approach}

In this research we have selected a reference architecture framework (AF) obtained by generalising several other AFs and thus potentially expressive enough to contain all the elements necessary to achieve EM integration using EA artefacts. This AF is GERAM (Generalised Enterprise Reference Architecture and Methodology), described in ISO 15704:2005 [16]. Among others, GERAM has been used in practice to guide EA projects [17], assess other enterprise AFs [18-20] and build a structured repository of AF elements for a project management decision support system [21].

The main component of the reference architecture of GERAM (called GERA) is a MF containing an extensive set of aspects including life cycle, management, organisation, human and decision, corresponding to various stakeholder concerns [22]. A subset of GERA has been used as a modelling formalism in the creation of a life cycle-based business model as subsequently shown in this paper. For more details on GERAM and GERA see [16]. 
The paper argues that EA can provide an overarching and life cycle-based approach in setting up and operating an EM project aiming to produce an EMS in an integrated and coherent manner in relation to the host organisation (VO), $\mathrm{CN}$ participants and other relevant external entities. To illustrate this approach, the researcher has used a meta-methodology, or a 'method to build methods' applicable for specific types of EA tasks (projects), based on an original approach abiding by EA principles. The meta-methodology, tested in several case studies [21, 23, 24] employs a set of steps and sub-steps. In the first step, the user is prompted to create a list containing entities of interest to the project in question, including project participants, target entities (e.g. the organisations / systems being created) and importantly, the project itself. The second step comprises the creation of business models showing the relations between the previously listed entities in the context of their lifecycles, i.e. illustrating how entities influence each other within each life cycle phase. The third step assists the user to infer the set of project activities by reading and interpreting the previous representations for each life cycle phase of the project and other target entities. The resulting activities are then decomposed to a level deemed suitable for the intended audience. Due to its scope and to space limitations, the paper will cover only the first and second meta-methodology steps, focusing in particular on the benefits of creating a business model in the context of the life cycles of all relevant participant entities. For more details on all the meta-methodology steps see [24].

\section{Application to the Environmental Management Project}

In this case, the meta-methodology deliverables are various models of the EM project and the EMS, created in a life cycle context. The $\mathrm{CN}$ participants, the VO, and other relevant entities (e.g. organisations, agencies, laws etc) that need to be involved in the EM project and/or influence the resulting EMS are to be included in the entity list (first step of the meta-methodology). Thus, their influence will be taken into account throughout the life cycles of the EM project and the EMS. An important prerequisite for EM integration into the organisation is thus fulfilled. The meta-methodology assists in creating new knowledge (in this case, how to go about setting up and operating the EM project and the EMS) based on 'context expertise', i.e. the knowhow of running the businesses including corporate culture, relations with suppliers, clients, authorities etc, typically available at middle and top management levels. The involvement of these roles in the methodology creation process establishes the conditions for management buy-in, support for the upcoming EM project and for the early involvement of the EA approach and department (if present) in the EM project. This will create the best conditions for the integrated development of the EMS.

Proposed members in the entity list (the first step) are the CN participants, the VO set up by the CN, the VO's EMS, the EM project (to build that EMS), environmental reports, NGOs, the government, Environmental Protection Agency (EPA), EM principles (e.g. 2R, TBL), EM laws, EM standards, EM frameworks, assessment and reporting frameworks, social responsibility standards, Quality Standards and EM consultants. As mentioned, the MF of GERA is adopted here as the most suitable to provide a formalism for the (mandatory) life cycle and other selected aspects. 
In this case, the future (TO-BE) state is incremental and based on the present (ASIS) state - i.e. no radical redesign is recommended when setting up an EMS. This requires the AS-IS state to be represented for all aspects. While there is no tangible advantage in showing separate AS-IS and TO-BE states in the business model, it is useful to do so in the decisional / organisational structure. This is because here it is imperative to clearly show where and how the functions of the EMS interact with the existing system so as to ascertain the degree of integration and effects of the EMS on the decisional and organisational structure of the VO. Separate TO-BE decisional / organisational models also help define various future scenarios.

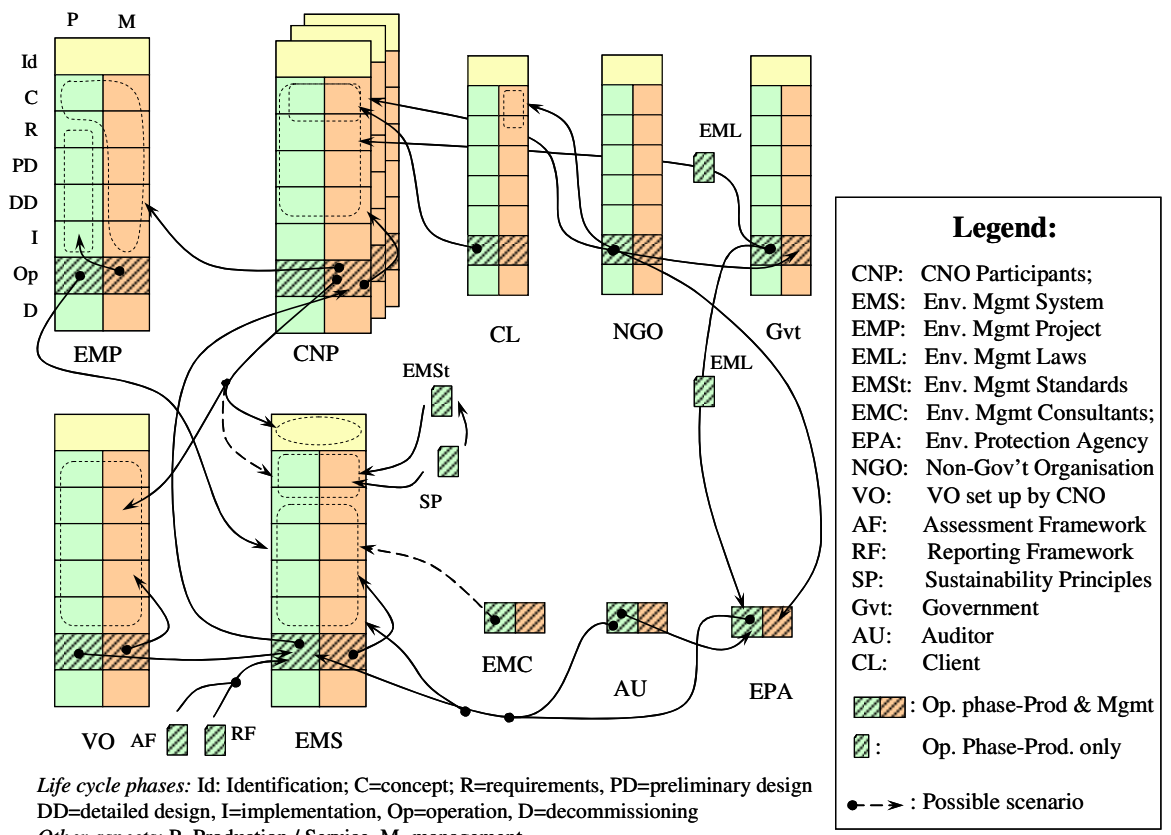

$\mathrm{DD}=$ detailed design, $\mathrm{I}=$ implementation, $\mathrm{Op}=$ operation, $\mathrm{D}=$ decommissioning Other aspects: $\mathrm{P}=$ Production / Service, $\mathrm{M}=$ management

Fig. 1. Business model showing relations of relevant entities in the context of their life cycles

The business model is constructed (in step two) based on context knowledge (often tacit and requiring eliciting by the meta-methodology facilitator) owned by stakeholders, i.e. $\mathrm{CxO}$, enterprise architect, top management, etc. A possible result is shown in Fig. 1. As can be seen, the relations between the relevant entities can be explicitly represented at each life cycle phase. Note that some entities' life cycle representation has been reduced to the phase(s) relevant for the EM project and the EMS. For example, we are only interested in the Operation life cycle phase of Auditors, EM assessment / reporting frameworks, EM consultants. The figure shows the relations between the CN participants, the VO, the EM project and the VO's future EMS, thus facilitating a common understanding, building consensus and representing what needs to be done, step by step, first at a high level and then more detailed as necessary. 


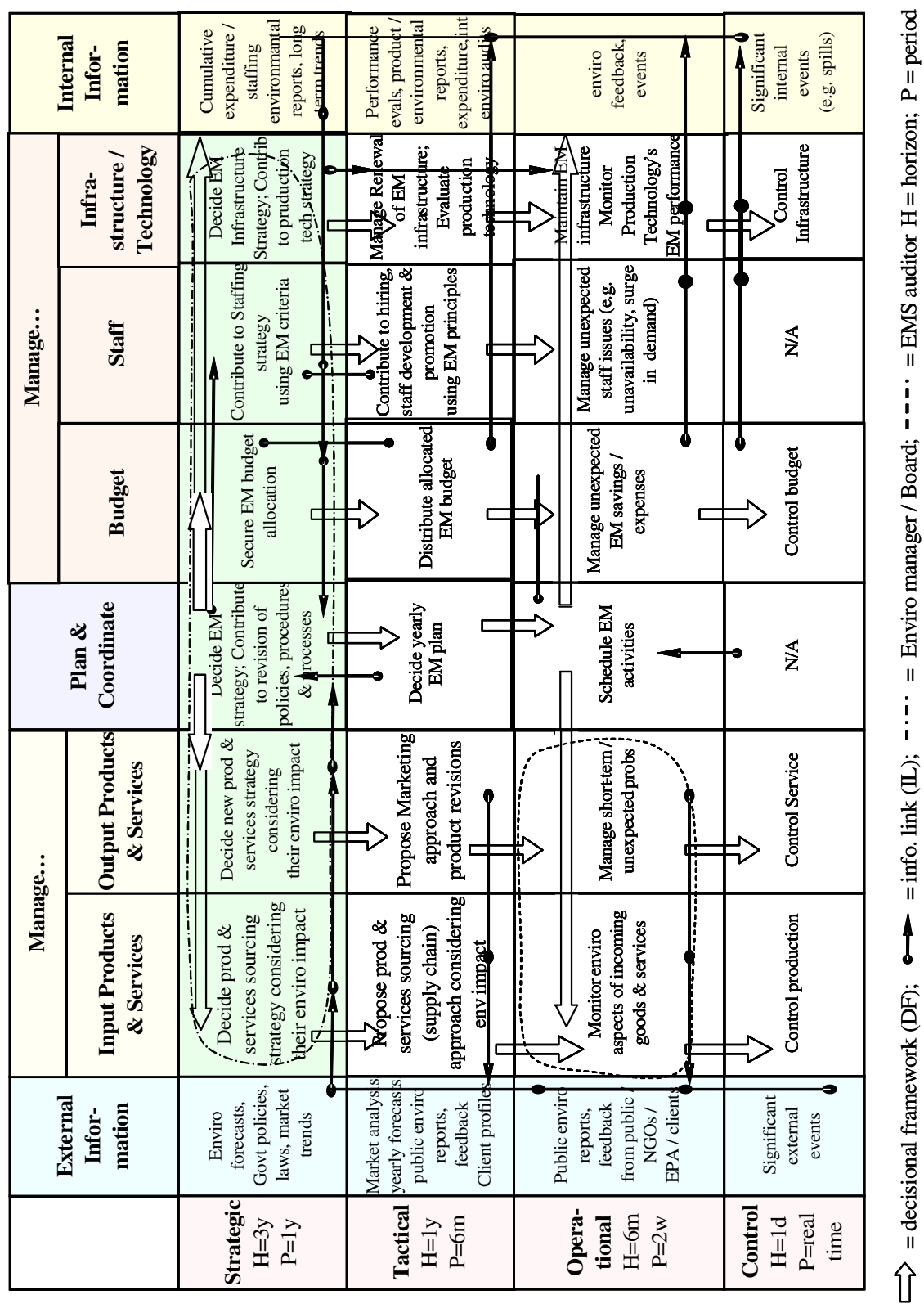

Fig. 2. Sample GRAI Grid [25] for EM integration in the VO (roles shown by dashed lines)

For example, the EMS is built by the EM project (EMP), with the possible involvement of consultants (EMC). The CN participants are lobbied by NGOs and must abide by EM laws (EML). Auditors (AU) perform certification audits (during 
the design of the EMS) or surveillance audits (to check if the EMS is still compliant). The EPA looks into the EMS operation / receives information from external auditors.

Importantly, the EMS should be able to redesign itself (arrow from its Mgmt operation to its other life cycles) to a certain extent and thus remain agile in the face of moderate EM regulation and market changes. Reaction to major changes will however be delegated to the upper company management via an EM project (EMP) (arrows from CN participants (CNP) management operation to EMP and from EMP operation to EMS life cycles). The arrow from the operation management side of the EMS to CNP management reflects the requirement to integrate (e.g. via partial redesign / co-design) CN participants' management to the VO's EMS functions. Such inter-relations are detailed in the next meta-methodology steps as controls and inputs to activities, decision frameworks, etc.

The influences of other entities on the EMS and the EMP can also be interpreted as stakeholder concerns that translate in particular areas of interest being modelled and addressed. For example, the client may want to know how the mission and vision of the CN participants (the Concept area of CNP in Fig. 1) address its environmental concerns, and the government (Gvt) will want to ensure that the Company abides by the public environmental concerns expressed in EM laws.

Note that in order to limit the complexity of the figure, relations between entities that are not relevant to the task at hand have been omitted (e.g. the general setup of the VO by the CNO, or most relations between government, auditors and EPA, etc).

Models of the AS-IS and several potential TO-BE decisional and organisational aspects have also been constructed. For example, Fig. 2 shows in a simplified form (using the GRAI-Grid [25] formalism) a possible TO-BE decisional / organisational structure of an EMS seen as an integrated add-on of the VO management system. This approach enables the organisation to manage, benchmark and improve its environmental performance in an integrated manner (i.e. taking into account all relevant areas in a cross-departmental approach).

The space available for this paper does not allow a detailed presentation. In-depth descriptions and additional models (including activity models of the third metamethodology step) are available in [26].

\section{Conclusions and Further Work}

Currently, businesses (including those participating in CNs) do not appear to achieve the maximum benefits from implementing and operating an EMS internally and/or in the VOs they create. Firstly, there seems to be a lack of integration of the EM initiative with the business, especially at the strategic level. Thus, the management cannot take full advantage of the knowledge present in the environmental reporting mainly due to wrong format and/or level of aggregation. Secondly, an EMS needs to be driven internally and permeate all business areas in a consistent manner in order to produce organisational culture change, hence lasting effects. This paper has argued for and tried to show (at high level due to space limitations) that such needs are best addressed from an EA perspective. Thus it appears that EA can provide the necessary artefacts and the prerequisites for a coherent, cross-departmental and culture-changing approach. This promises business sustainability and profitability in the long term for $\mathrm{CN}$ members but also agility and competiveness for the VOs created by CNs. 


\section{References}

1. Elkington, J.: Cannibals with Forks: The Triple Bottom Line of $21^{\text {st }}$ Century Business (1998)

2. Blackburn, W.R.: The Sustainability Handbook. EarthScan Publishers, Cornwall (2007)

3. UN World Commission on Environment and Development. Our Common Future (Brundtland Report). Oxford University Press, Oxford (1987)

4. Camarinha-Matos, L., et al.: Collaborative networked organizations - Concepts and practice in manufacturing enterprises. Computers and Industrial Engineering 57(1), 46-60 (2009)

5. Nilsson, I.: Integrating Environmental Management to Improve Strategic DecisionMaking. Chalmers University of Technology, Götteborg (2001)

6. Molloy, I.: Environmental Mgmt Systems and Info Mgmt, Strategic-Systematical Integration of Green Value Added. In: Gómez, J.M., et al. (eds.) ITEE 2007 - (3 ${ }^{\text {rd }}$ Int. ICSC Symp.) (2007)

7. Coglianese, C., Nash, J. (eds.): Regulating from the Inside: Can Environmental Management Systems Achieve Policy Goals? RFF Press (2001)

8. ISO, ISO 14001: Environmental management systems - Requirements with guidance for use. International Standards Organisation (2004)

9. Willard, B.: The Sustainability Advantage: Seven Business Case benefits of a Triple Bottom Line. New Society Publishers, Gabriola Island (2002)

10. Clayton, A., Redcliffe, N.: Sustainability - A Systems Approach. Earthscan Ltd. (1998)

11. Upham, P.: An assessment of the Natural Step theory of sustainability. Journal of Cleaner Production 8(6), 445-454 (2000)

12. TNEP: The Engineering Sustainable Solutions Program Whole Systems Design Suite. The Natural Edge Project, TNEP (2007)

13. Hunkeler, D. (ed.): Life-cycle Management. Society of Environmental Toxicology etc. (2004)

14. EPA: Management Tools. Environmental Protection Agency, South Australia (2008)

15. GRI: Sustainability Reporting Guidelines, in Sustainability Reporting Framework. Global Reporting Initiative (ed.): Global Reporting Initiative (2002)

16. ISO/IEC: Annex C: GERAM, in ISO/IS 15704:2000/Amd1: 2005: Industrial automation systems - Requirements for enterprise-reference architectures and methodologies (2005)

17. Bernus, P., Noran, O., Riedlinger, J.: Using the Globemen Reference Model for Virtual Enterprise Design in After Sales Service, in Globemen. In: Karvoinen, I., et al. (eds.) VTT Symposium, vol. 224. Helsinki, Finland, pp. 71-90 (2002)

18. Noran, O.: A Mapping of Individual Architecture Frameworks on GERAM. In: Bernus, P., Nemes, L., Schmidt, G. (eds.) Handbook of Enterprise Architecture, pp. 65-210. Springer, Heidelberg (2003)

19. Noran, O.: An Analytical Mapping of the C4ISR Architecture Framework onto ISO15704 Annex A (GERAM). Computers in Industry 56(5), 407-427 (2005)

20. Saha, P.: A Synergistic Assessment of the FEAF against GERAM (ISO15704:2000 Annex A). In: Saha, P. (ed.) Enterprise Systems Architecture in Practice, pp. 1-17. IDEA Group, USA (2007)

21. Noran, O.: Discovering and modelling Enterprise Engineering Project Processes. In: Saha, P. (ed.) Enterprise Systems Architecture in Practice, pp. 39-61. IDEA Group, USA (2007)

22. ISO/IEC: ISO/IEC 42010:2007: Recommended Practice for Architecture Description of Software-Intensive Systems (2007)

23. Noran, O.: Using Reference Models in Enterprise Architecture: An Example. In: Fettke, P., Loos, P. (eds.) Reference Modeling for Business Systems Analysis, pp. 141-165. Idea Group, Hershey (2006)

24. Noran, O.: A Meta-methodology for Collaborative Networked Organisations: Creating Directly Applicable Methods for Enterprise Engineering Projects. VDM, Saarbrücken (2008)

25. Doumeingts, G., Vallespir, B., Chen, D.: GRAI Grid Decisional Modelling. In: Bernus, P., Mertins, K., Schmidt, G. (eds.) Handbook on Archs of IS, pp. 313-339. Springer, Heidelberg (1998)

26. Noran, O.: Engineering the Sustainable Business: An Enterprise Architecture Approach. In: Doucet, G., Gotze, J., Saha, P. (eds.) Coherency Management: Architecting the Enterprise for Alignment, Agility, and Assurance, Int. Enterprise Architecture Institute, pp. 179-210 (2009) 